\title{
Limits on Discrete Modulated Signals
}

\author{
Alan C. Bovik, Fellow, IEEE, Joseph P. Havlicek, Member, IEEE, \\ Mita D. Desai, Senior Member, IEEE, and David S. Harding, Student Member, IEEE
}

\begin{abstract}
We develop theorems of a general nature that apply to the analysis of AM-FM signals of the form $a(m) \exp [j \phi(m)]$ or $a(m) \cos [\phi(m)]$ and to their behavior both in linear systems and in simple nonlinear systems comprised of products of linear elements. Such product-systems include interesting nonlinear demodulation operators, such as the Teager-Kaiser operator. Expressions for the approximate system responses to AM-FM signals are derived by making an analogy to the eigenfunction interpretation of sinusoids in linear systems; for the case of sinusoidal signals, the approximations are exact. These expressions are collectively called quasieigenfunction approximations (QEA's). For nonsinusoidal AM-FM signals, the approximations have errors that are tightly bounded by functionals that express the smoothness of the AM and FM information signals and the durations of the involved system impulse responses. The bounds are independent of the bandwidths of the AM and FM functions. Two general applications are considered. First, the approximations are found to be useful for analyzing discretetime nonlinear energy operators, including the Teager-Kaiser operator. Next, the approximation theorems lead to the selection of an optimal class of bandpass filters for use in a discrete multiband AM-FM demodulation system. The filter class selected is optimal in the sense of achieving the lower bound of a novel discrete uncertainty principle.
\end{abstract}

\section{INTRODUCTION}

W E DEVELOP useful approximations to the responses of general discrete linear systems, simple nonlinear systems comprised of products of linear elements, and in particular, certain discrete nonlinear AM-FM energy operators to complex signals of the form

$$
c(m)=a(m) \exp [j \phi(m)]
$$

and their real-valued counterparts

$$
r(m)=a(m) \cos [\phi(m)]
$$

where $a: Z \rightarrow \boldsymbol{R}$ are samples of a continuously differentiable amplitude-modulation (AM) function $a(t)$, and $\phi: Z \rightarrow R$ are samples of a continuously twice-differentiable frequencymodulation (FM) function $\phi(t)$. We also supply tight bounds on these approximations that are expressed in terms of the smoothness of $a(t)$ and $\phi(t)$, as measured by certain (Sobolev) smoothness norms, as well as in terms of the duration of the involved linear system function(s).

Manuscript received August 28, 1995; revised December 3, 1996. This work was supported in part by a grant from the Texas Advanced Research Projects Agency and by the Air Force Office of Scientific Research, Air Force Systems Command, USAF, under Grant F49620-93-1-9307. The associate editor approving the review of this paper and approving it for publication was Dr. Nurgun Erdol.

A. C. Bovik, J. P. Havlicek, and D. S. Harding are with the Department of Electrical and Computer Engineering, The University of Texas at Austin, Austin, TX 78712-1084 USA.

M. D. Desai is with the Division of Engineering, The University of Texas at San Antonio, San Antonio, TX 78729 USA.

Publisher Item Identifier S 1053-587X(97)02643-3.
AM and FM functions of the form (1) and (2) are gaining popularity as effective and practical tools for modeling nonstationary, yet locally coherent structures in speech signals, images, and other variably dimensional signals. For example, these models have been successfully used in the analysis of textured images when combined with Gabor wavelet image decompositions [1]-[3] and/or certain nonlinear energy operators [4]. Models of the form (1) have also been extensively applied to the analysis of speech formation with good success [5]-[8].

In these applications, it is often desirable to pass the signal of interest through a linear system, such as a bandpass filter [3], in order to extract and separate local frequency (modulation) structures that the model (1), (2) captures. In Section II, we find new approximations for the responses of two classes of systems to the inputs (1), (2). The first class of system is arbitrary discrete linear systems having finite-energy impulse response; the second class of (nonlinear) system is defined by products of linear systems. We state and prove theorems in each case that bound the error between the approximation and the actual responses. These results are applied in Section III to establish theorems characterizing the validity of similar approximations to the responses of certain simple nonlinear AM-FM (Teager-Kaiser-type) energy operators [4]-[10]. Each result is presented for the complex AM-FM input $a(m) \exp [j \phi(m)]$ with an analogous result for the real input $a(m) \cos [\phi(m)]$ given as a corollary.

In Section IV, we apply the results further in the analysis of a discrete multiband AM-FM demodulation scheme. Such systems are effective for extracting sophisticated AM-FM signal components in the presence of noise, multicomponents, or other signal artifacts [11], [12]. As a byproduct of the analysis, an optimal class of bandpass filters is derived for demodulation purposes.

\section{ApProximation to Linear System Response AND PRODUCTS OF LINEAR System RESPONSES}

In this section, theorems are given that motivate new approximations to the responses of arbitrary square-summable discrete linear systems to inputs of the form (1), (2) as well as to (nonlinear) products of such responses. The results are general; however, in later sections, we will be most interested in application systems that involve either simple differencers or bandpass systems with impulse responses of the form

$$
h(m)=w(m) \exp \left(j \omega_{c} m\right)
$$

where $w(m)$ is a real-valued lowpass function. Both differencers and bandpass filters are used in the study of discrete multiband demodulation systems in Section IV. 


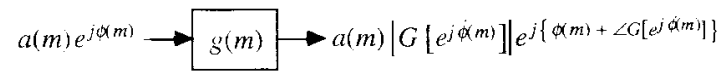

Fig. 1. Depiction of basic QEA for complex AM-FM signals.

\section{A. Approximate Linear System Response}

In the first general result, we approximate the response

$$
d(m)=g(m) * c(m)=\sum_{p \in \boldsymbol{Z}} g(p) c(m-p)
$$

of an arbitrary square-summable discrete linear system $g: Z \rightarrow$ $C$ to an input of the form (1). The approximation is given by

$$
\begin{aligned}
\hat{d}(m) & =c(m) \cdot G\left[e^{j \dot{\phi}(m)}\right] \\
& =a(m)\left|G\left[e^{j \dot{\phi}(m)}\right] \exp \right|\left\{j \phi(m)+\angle G\left[e^{j \dot{\phi}(m)}\right]\right\}
\end{aligned}
$$

where we denote $\dot{\phi}(m)=\left.(d / d t) \phi(t)\right|_{t=m}$ and where

$$
G\left(e^{j \omega}\right)=\sum_{m \in \boldsymbol{Z}} g(m) e^{-j \omega m}
$$

is the frequency response of the linear system $g(m)$. The approximation is also depicted in Fig. 1.

Hereafter, we shall use the notation $g(m) \leftrightarrow G\left(e^{j \omega}\right)$ to denote a Fourier transform pair. The approximation (5), when valid, has a powerful natural application for the analysis of discrete linear systems that have AM-FM inputs modeled by (1). The approximation has a general form that is analogous to the form of the response of the system to a monochromatic signal

$$
c(m)=a_{0} \exp \left(j \omega_{0} m\right)
$$

except that in (5), the argument of the system function $G(\cdot)$ is time varying. Indeed, for the case (7) of the monochromatic signal, the approximation (5) is, of course, exact: $d(m)=$ $\hat{d}(m)$. Hence, we refer to this approximation (and its relatives) as quasieigenfunction approximations (QEA's). When the signal $c(m)$ is not monochromatic, there will always be an error in the approximation; however, this error may be small and can, in fact, be bounded, as will be established by Theorem 1 and Lemma 1. Denote

$$
a_{\max }=\sup _{m \in \boldsymbol{Z}}|a(m)| .
$$

In certain applications such as image analysis, where $a(m)$ can be used to model contrast, it may be assumed that $a_{\max }=1$. Note again that $a(m)$ and $\phi(m)$ are regarded as samples of continuous functions, the derivatives of which appear in the integrals. Finally, we shall denote $p^{+}=\max \{0, p\}$ and $p^{-}=\min \{0, p\}$.

Theorem 1: Let $\varepsilon_{d}(m)=|d(m)-\hat{d}(m)|$, where $d(m), \hat{d}(m)$ are given by (4) and (5). Then

$$
\varepsilon_{d}(m) \leq \sum_{\substack{p \in Z \\ p \neq 0}}|g(p)| \int_{m-p^{+}}^{m-p^{-}}|\dot{a}(v)|+a_{\max }|p||\ddot{\phi}(v)| d v .
$$

Proof: All proofs are given in the Appendix.

Several observations may immediately be made regarding the bound in (8). Clearly, the error bound is reduced if the derivative magnitudes $|\dot{a}(r)|$ and $|\ddot{\phi}(r)|$ are uniformly small; this is further made clear in Lemma 1. However, it is also clear that the bound will be reduced when the duration of $g(p)$ is small; in subsequent developments, we will measure the duration (normalized by the square root of energy in the case that $g(m)$ is not unit-energy) of a filter $g(m)$ using the general-purpose energy moment functionals

$$
\begin{aligned}
\Delta_{k}(g) & =\left[\sum_{m \in Z} m^{2 k}|g(m)|^{2}\right]^{1 / 2} \\
& =\sqrt{\frac{1}{2 \pi} \int_{-\pi}^{\pi}\left|\frac{d^{k}}{d \omega^{k}} G\left(e^{j \omega}\right)\right|^{2} d \omega .}
\end{aligned}
$$

Note that a bandpass filter $h(m)$, as given in (3), and its lowpass equivalent $w(m)$ have the same durations (9): $\Delta_{k}(w)=$ $\Delta_{k}(h)$.

The bound in (8) clarifies the interplay between the duration of the filter $g(m)$ and the local behavior of $|\dot{a}(r)|$ and $|\ddot{\phi}(r)|$. Thus, (8) is a useful numerical tool for examining the efficacy of (5) when analyzing signals for which the integrals of $|\dot{a}(r)|$ and $|\ddot{\phi}(r)|$ may be easily evaluated or for which they may be numerically calculated with some effort. However, Theorem 1 does not supply a design procedure for the filter $g(m)$ (to isolate a component of the form (1) from the remainder of a signal, for example), nor does it make explicit the individual roles of the filter and the AM-FM functions for approximating the responses of unspecified signals.

Note that (8) implies that the error does not depend on $|g(0)|$; indeed, if the filter is an impulse $|g(m)|=K \delta(m)$, then the error is zero. For even symmetric filter magnitudes, the bound (8) becomes

$$
\varepsilon_{d}(m) \leq \sum_{p>0}|g(p)| \int_{m-p}^{m+p}|\dot{a}(v)|+a_{\max } p|\ddot{\phi}(v)| d v .
$$

Lemma 1 yields a bound expressed in terms of products of measures of the filter duration, as expressed by (9), with global measures of the smoothness of $a(t)$ and $\dot{\phi}(t)$, as expressed by derivative functionals, or Sobolev norms, of the form

$$
\|a\|_{\mathcal{D}^{k}}=\int_{\boldsymbol{R}}\left|a^{(k)}(u)\right| d u
$$

where $a^{(k)}(u)=d^{k} a(u) / d u^{k}$. In particular, we will make use of the norms $\|a\|_{\mathcal{D}^{1}}$ and $\|\phi\|_{\mathcal{D}^{2}}$. Since only finite bounds are of interest, these norms may be assumed finite, or equivalently, that the AM-FM functions $a(t)$ and $\phi(t)$ exist in Sobolev spaces of degrees 1 and 2, respectively. Under this assumption, the integrals in (8) and (10) are guaranteed to converge as well.

We will also encounter the constant

$$
\gamma=\sum_{\substack{p \in Z \\ p \neq 0}} \frac{1}{p^{2}}=\frac{\pi^{2}}{3}=3.2899 \cdots
$$

which is twice Riemann's Zeta function with argument 2. Note that $\sqrt{\gamma} \approx \pi / \sqrt{3}=1.8138$.

Lemma 1: Let $\varepsilon_{d}(m)=|d(m)-\hat{d}(m)|$ as before. Then

$$
\varepsilon_{d}(m) \leq \sqrt{\gamma}\left[\Delta_{1}(g) \cdot\|a\|_{\mathcal{D}^{1}}+a_{\max } \Delta_{2}(g)\|\phi\|_{\mathcal{D}^{2}}\right] .
$$

Proof: See the Appendix. 


$$
a(m) \cos [\phi(m)] \rightarrow h(m) \rightarrow a(m)\left|H\left[e^{j \dot{\phi}(m)}\right]\right| \cos \left\{\phi(m)+\angle H\left[e^{j \phi(m)}\right]\right\}
$$

Fig. 2. Depiction of basic QEA for real AM-FM signals.

In Lemma 1, the approximation error is explicitly bounded by expressions of the overall duration of the filter $g(m)$ and by the global smoothness (or lack thereof) of $a(t)$ and $\dot{\phi}(t)$. Indeed, the bound does not include dependence on time. Although it is shown in the proof that (11) is bounded below by (8), the bound (11) is still tight: Note that as $c(m)$ becomes monochromatic, both $\|a\|_{\mathcal{D}^{1}}$ and $\|\phi\|_{\mathcal{D}^{2}}$ vanish. The bound (11) also has the advantage of simplicity of interpretation: The filter and the AM-FM functions are represented independently. Later, this will yield design criteria in a multiband demodulation system. Of course, for signals of infinite length, the bound will often be infinite, thus limiting its usefulness compared to (8) in such cases.

Example 1-Chirp Signal: The constant-amplitude complex chirp signal $c(m)=A \exp \left[j\left(B m^{2}+C m+D\right)\right]$, where $A, B, C$, and $D$ are constant, is of interest in many applications. Assuming that the signal $c(m)$ is applied to the square-summable discrete linear system $g(m) \leftrightarrow G\left(e^{j \omega}\right)$, the QEA is then

$\hat{d}(m)=A \exp \left[j\left(B m^{2}+C m+D\right) \cdot G\{\exp [j(2 B m+C)]\}\right.$.

The error in this approximation cannot be usefully bounded by (11) since $\|\phi\|_{\mathcal{D}^{2}}=\infty$. However, (8) yields a convenient bound. Indeed, letting $f(m)=\sqrt{|g(m)|}$, we find $\varepsilon_{d}(m) \leq$ $2 A B \Delta_{1}(f)$, which is both simple and suggestive. The error bound of the QEA for a chirp signal is linear with respect to both the signal amplitude and to the chirp sweep rate; the bound is also independent of time $m$ and, hence, also of the local frequency. From this, we may see that the error bound in the approximation is primarily governed by the rate of change of the local AM-FM signal frequencies and by the filter duration.

Next, we approximate the response

$$
s(m)=h(m) * r(m)=\sum_{p \in Z} h(p) r(m-p)
$$

of an arbitrary real-valued, square-summable discrete linear system $h: Z \rightarrow \boldsymbol{R}$ to a real AM-FM input $r(m)$ of the form (2). The QEA is

$$
\hat{s}(m)=a(m) \cdot\left|H\left[e^{j \dot{\phi}(m)}\right]\right| \cos \left\{\phi(m)+\angle H\left[e^{j \dot{\phi}(m)}\right]\right\}
$$

where $h(m) \leftrightarrow H\left(e^{j \omega}\right)$, as depicted in Fig. 2. The bound for this analogous result for real filters and real AM-FM signals is supplied by the following Corollary to Theorem 1 and Lemma 1.

Corollary 1: Let $\varepsilon_{s}(m)=|s(m)-\hat{s}(m)|$, where $s(m), \hat{s}(m)$ are given by (12) and (13). Then

$$
\begin{aligned}
\varepsilon_{s}(m) & \leq \sum_{\substack{p \in Z \\
p \neq 0}}|h(p)| \int_{m-p^{+}}^{m-p^{-}}|\dot{a}(v)|+a_{\max }|p||\ddot{\phi}(v)| d v \\
& \leq \sqrt{\gamma}\left[\Delta_{1}(h) \cdot\|a\|_{\mathcal{D}^{1}}+a_{\max } \Delta_{2}(h)\|\phi\|_{\mathcal{D}^{2}}\right] .
\end{aligned}
$$

Proof: See the Appendix.
These results are quite novel in the analysis of discrete AMFM signals, although results analogous to Corollary 1 have been developed for continuous AM-FM signals in [11]. The bounds on the errors $\varepsilon_{d}(m), \varepsilon_{s}(m)$ become small whenever both AM-FM functions $a(t), \dot{\phi}(t)$ are sufficiently smooth (locally and/or globally). The bounds that require global smoothness, which are expressed as Sobolev norms, have magnitudes modulated by the filter durations. A rapidly decaying filter will also control the size of the local bounds, which are expressed as sums of local smoothness measures distance-weighted by the filter.

\section{B. Numerical Results}

We now further establish the usability of the basic approximations and error bounds that have been developed thus far. This is accomplished through simulations involving a generic parametrized input AM-FM signal. By varying the parameters, we also find some intuition into the validity of the approximations.

In all of the simulations in this and later sections, we shall assume a (complex) input AM-FM signal of the form (1), where the AM function is a Gaussian

$$
a(m)=\exp \left(-m^{2} / 2 \sigma_{a}^{2}\right)
$$

and the FM function is the product of a Gaussian and a quadratic

$$
\phi(m)=\exp \left(-m^{2} / 2 \sigma_{\phi}^{2}\right) \cdot\left(B m^{2}+C m\right) .
$$

In (15), the constants $B$ and $C$ are chosen such that the derivative of the quadratic (chirp) component of the phase takes initial value $\omega_{i}=2 B m_{i}+C$ and final value $\omega_{f}=$ $2 B m_{f}+C$. Here, $m_{i}$ and $m_{f}$ are the initial and final instants of the interval over which the response is plotted. Specifying $\omega_{i}$ and $\omega_{f}$ is a simple method of fixing the approximation range of instantaneous frequencies contained in the signal.

We will suppose that the AM-FM signal described by (14) and (15) is passed through a filter with impulse response a Gabor function with center frequency $\omega_{g}$ (radians)

$$
g(m)=K \cdot \exp \left(-m^{2} / 4 \sigma_{g}^{2}\right) \cdot \exp \left(j \omega_{g} m\right)
$$

where $K$ is selected such that $g(m)$ has unit energy (unit $l_{2^{-}}$ norm). By varying the parameters of both input signal and filter, we will assess the behavior of the error bound (8). Fig. 3(a) plots the bound for $\omega_{i}=0.4 \pi \mathrm{rad}, \omega_{f}=0.6 \pi \mathrm{rad}$, $\sigma_{a}=1024$ (samples), and four values of the phase duration parameter $\sigma_{\phi}$. The filter center frequency was selected to be $\omega_{g}=\pi / 2 \mathrm{rad}$ and the bandwidth to be one octave $\left(\sigma_{g}=1.59\right)$. As expected, the error (hence, the bound) generally falls as $\sigma_{\phi}$ is increased; moreover, the bound becomes nearly constant as $\sigma_{\phi}$ becomes large (hence, the phase nearly quadratic), which is not unexpected in view of the result in Example 1. For smaller values of $\sigma_{\phi}$, the Gaussian phase term begins to dominate. It will be observed that in all cases, the bound is quite small.

Fig. 3(b) shows the behavior of the bound (8) as the frequency range $\Delta \omega=\omega_{f}-\omega_{i}$ was varied. Here, the other signal and filter parameters remained the same, with the phase duration parameter fixed at $\sigma_{\phi}=4096$ (samples). For $\Delta \omega$ small, the overall signal becomes more narrowband-more eigenfunction-like. The bound agrees with this observation and falls accordingly. 


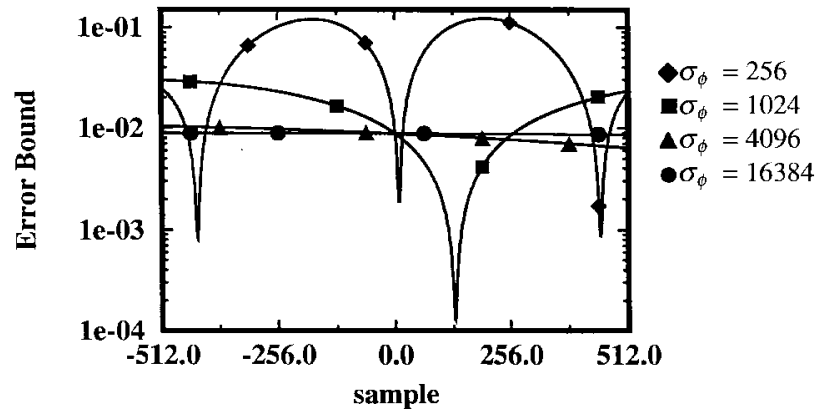

(a)

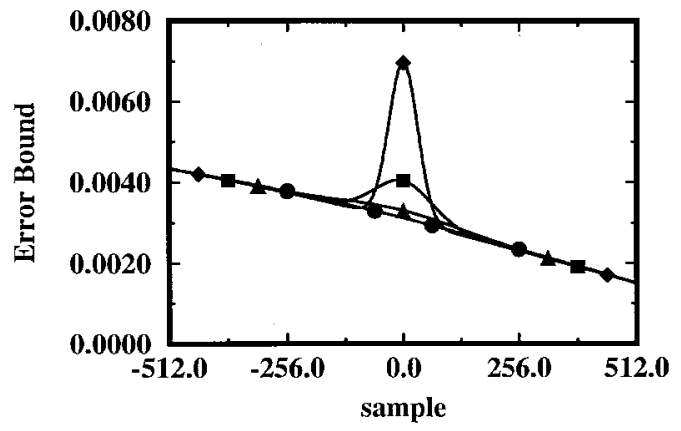

(c)

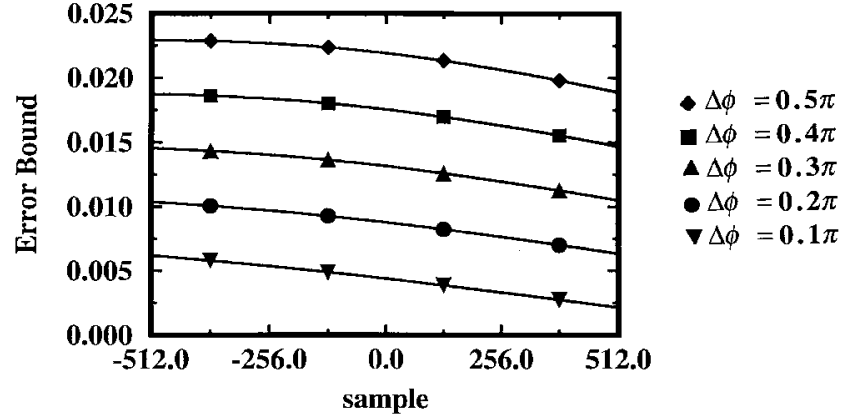

(b)

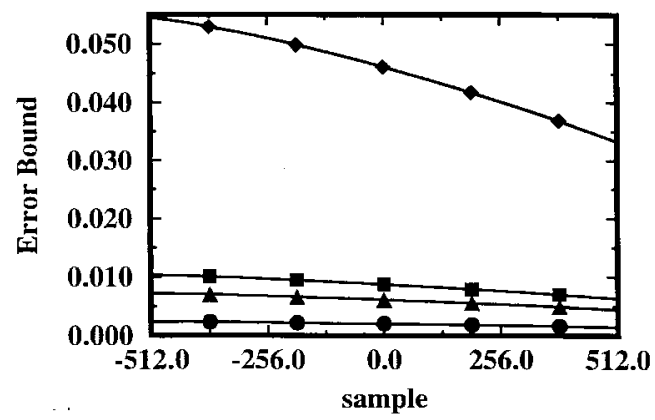

(d)

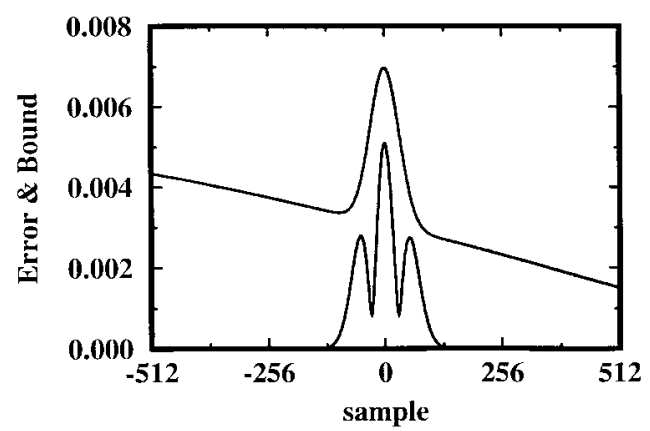

(e)

Fig. 3. QEA error bound (8) as a function of (a) the duration of the Gaussian phase term, (b) the duration of the quadratic phase term, (c) the duration of the Gaussian amplitude term, and (d) the duration of the filter: $\bullet \sigma_{g}=3.09$, $\sigma_{g}=1.59, \boldsymbol{\Delta} \sigma_{g}=1.38$, $\bullet \sigma_{g}=0.88$. Finally, (e) depicts true error and error bound simultaneously.

To illustrate the effect of amplitude modulation on the bound, the phase and filter parameters were fixed and the amplitude duration parameter $\sigma_{a}$ allowed to vary. In this example, the instantaneous frequency range was changed to $\Delta \omega=0.1 \pi \mathrm{rad}$ (from $\omega_{f}=0.45 \pi \mathrm{rad}$ to $\omega_{f}=0.55 \pi$ rad) simply to produce a more noticeable effect in the bound. The filter bandwidth was also increased to 1.5 octaves $\left(\sigma_{g}=\right.$ 1.378). The bound is seen in Fig. 3(c) to increase fairly quickly as the $\mathrm{AM}$ function is narrowed ( $\sigma_{a}$ reduced), although in all cases, it is quite small. However, some care may be needed in applying QEA's to short-duration input signals.

Next, the signal parameters were all held fixed and the filter duration varied, as shown in Fig. 3(d). The fixed parameters were $\Delta \omega=0.2 \pi, \sigma_{a}=1024$ samples, $\sigma_{\phi}=4096$ samples, and the filter c.f. $=\pi / 2 \mathrm{rad}$. As expected, the error decreased with filter duration in a predictable way. The bandwidths corresponding to $\sigma_{g}=3.09,1.59,1.38$, and 0.88 samples are $0.5,1.0,1.5$, and 2.0 octaves.
Finally, Fig. 3(e) plots the error bound (8) over the actual approximation error for all the same parameter values as Fig. 3(d) and the filter fixed at one octave. It is interesting to note the tracking of the error bound where the error rises.

As a second example, Fig. 4(a) plots the (real part of the) QEA $\hat{d}(m)$ given by (5), for another Gaussian-modulated chirp input. The input parameters were again $\Delta \omega=0.2 \pi$ and $\sigma_{s}=1024$ samples, only this time, $\sigma_{\phi}=1024$ samples. The filter was selected identical with that used in Figs. 3(a)-(c) and (e). The error depicted in Fig. 4(b) is small everywhere, dropping close to zero at some points. Fig. 4(c) shows the close tracking of the bound (8) near one such downward excursion in the true error.

\section{Approximate Product-System Response}

Next, we develop approximations to the products of linear system responses. This has immediate application, as shown in Section III, for the analysis of nonlinear systems that in- 


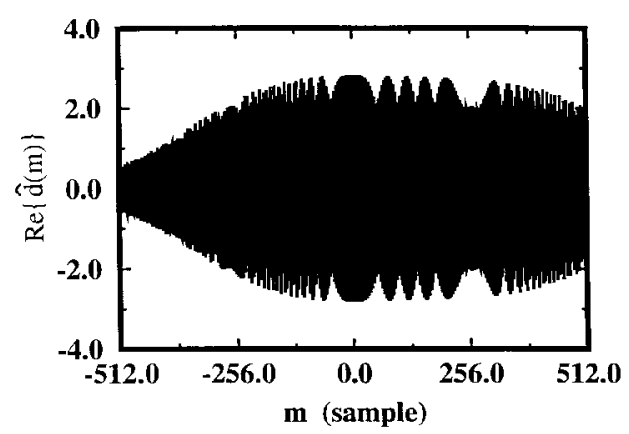

(a)

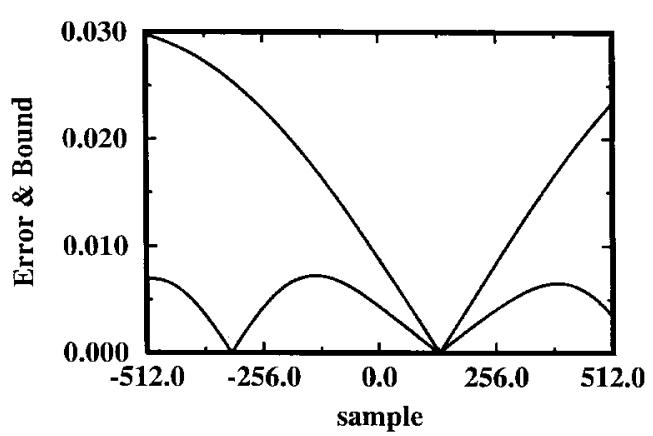

(b)

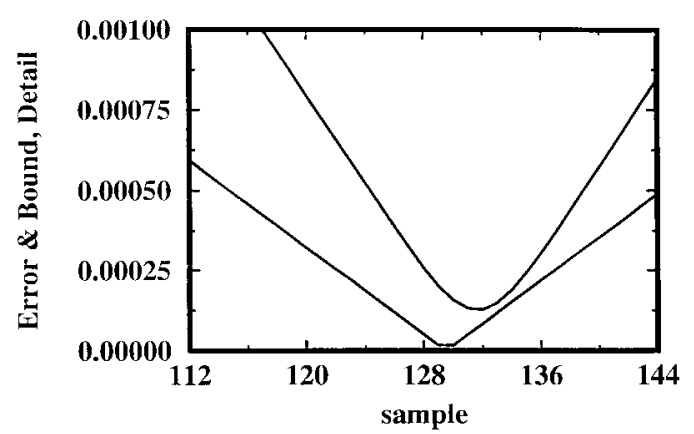

(c)

Fig. 4. QEA for Gaussian-modulated chirp signal. (a) Real part of the QEA. (b) Error and bound. (c) Detail of (b).

corporate square-law devices and other product nonlinearities. Denote the product of response and conjugate response

$$
b(m)=\left[g_{1}(m) * c(m)\right] \cdot\left[g_{2}(m) * c(m)\right]^{*}
$$

of arbitrary square-summable discrete linear systems $g_{1}: Z \rightarrow$ $C, g_{2}: Z \rightarrow C$ to an input of the form (1). The approximation is given by

$$
\hat{b}(m)=a^{2}(m) \cdot G_{1}\left[e^{j \dot{\phi}(m)}\right] G_{2}^{*}\left[e^{j \dot{\phi}(m)}\right]
$$

where $g_{k}(m) \leftrightarrow G_{k}\left(e^{j \omega}\right), k=1,2$. While the approximation (18) is not completely unexpected in view of the approximation (5) to (4) and the preceding bounds, it is not possible, unfortunately, to develop a useful bound on the error between (17) and (18) from Theorem 1 directly. However, the following Lemma, the proof of which makes use of elements of the proofs of previous results, does supply such a bound. Denote the $l_{1}$-norm of $g(m): \bar{g}=\Sigma_{p \in \boldsymbol{Z}}|g(p)|$.

Lemma 2: Let $e_{b}(m)=|b(m)-\hat{b}(m)|$, where $b(m), \hat{b}(m)$ are given by (17) and (18). Then

$$
\begin{aligned}
e_{b}(m) \leq & a_{\max } \sum_{\substack{p \in \boldsymbol{Z} \\
p \neq 0}}\left[\bar{g}_{2}\left|g_{1}(p)\right|+\bar{g}_{1}\left|g_{2}(p)\right|\right] \\
& \cdot \int_{m-p^{+}}^{m-p^{-}}|\dot{a}(v)|+a_{\max } \mid p\|\ddot{\phi}(v)\| d v \\
\leq & a_{\max } \sqrt{\gamma}\left\{\left[\bar{g}_{2} \Delta_{1}\left(g_{1}\right)+\bar{g}_{1} \Delta_{1}\left(g_{2}\right)\right]\|a\|_{\mathcal{D}^{1}}\right. \\
& \left.+a_{\max }\left[\bar{g}_{2} \Delta_{2}\left(g_{2}\right)+\bar{g}_{1} \Delta_{2}\left(g_{2}\right)\right]\|\phi\|_{\mathcal{D}^{2}}\right\} .
\end{aligned}
$$

Proof: See the Appendix.

The analogous result for real-valued AM-FM inputs applied to arbitrary real-valued square-summable discrete linear systems $h_{1}: Z \rightarrow \boldsymbol{R}, h_{2}: Z \rightarrow \boldsymbol{R}$ follows easily; the proof, which tracks that of Lemma 2 much in the same way that the proof of Corollary 1 follows Theorem 1, is omitted for brevity. Defining

$$
y(m)=\left[h_{1}(m) * r(m)\right] \cdot\left[h_{2}(m) * r(m)\right]
$$

we consider the approximation

$$
\begin{aligned}
\hat{y}(m)= & a^{2}(m) \cdot\left|H_{1}\left[e^{j \dot{\phi}(m)}\right]\right|\left|H_{2}\left[e^{j \dot{\phi}(m)}\right]\right| \\
& \cdot \cos \left\{\phi(m)+\angle H_{1}[\dot{\phi}(m)]\right\} \\
& \cdot \cos \left\{\phi(m)+\angle H_{2}[\dot{\phi}(m)]\right\}
\end{aligned}
$$

where $h_{k}(m) \leftrightarrow H_{k}\left(e^{j \omega}\right), k=1,2$.

Corollary 2: Let $e_{y}(m)=|y(m)-\hat{y}(m)|$, where $y(m)$ and $\hat{y}(m)$ are given by (21) and (22). Then

$$
\begin{aligned}
e_{y}(m) \leq & a_{\max } \sum_{\substack{p \in Z \\
p \neq 0}}\left[\bar{h}_{2}\left|h_{1}(p)\right|+\bar{h}_{1}\left|h_{2}(p)\right|\right] \\
& \cdot \int_{m-p^{+}}^{m-p^{-}}|\dot{a}(v)|+a_{\max }|p \| \ddot{\phi}(v)| d v \\
\leq & a_{\max } \sqrt{\gamma}\left\{\left[\bar{h}_{2} \Delta_{1}\left(h_{1}\right)+\bar{h}_{1} \Delta_{1}\left(h_{2}\right)\right]\|a\|_{\mathcal{D}^{1}}\right. \\
& \left.+a_{\max }\left[\bar{h}_{2} \Delta_{2}\left(h_{1}\right)+\bar{h}_{1} \Delta_{2}\left(h_{2}\right)\right]\|\phi\|_{\mathcal{D}^{2}}\right\} .
\end{aligned}
$$

Proof: The proof has been omitted for brevity.

Although the approximations in Lemma 2 and Corollary 2 are just products of previous approximations, it does not follow (without separate proof) that the product approximations are valid. In fact, we make the following general observation in passing. Suppose that $\hat{A}$ and $\hat{B}$ estimate some quantities $A$ 


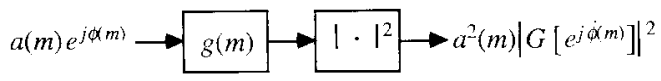

Fig. 5. QEA of the squared magnitude of a linearly-filtered complex AM-FM signal.

and $B$, respectively. Then, the supposition that $|\hat{A}-A|$ and $|\hat{B}-B|$ are small does not imply that

$$
|\hat{A} \hat{B}-A B|=|(\hat{A}-A)(\hat{B}+B)+(\hat{A}+A)(\hat{B}-B)| / 2
$$

is small since it depends on the magnitudes of all involved quantities. Indeed, it does not even imply that $\left|\hat{A}^{2}-A^{2}\right|$ is small!

\section{APPliCATion to NONLINEAR \\ AM-FM ENERGY OPERATORS}

We now explore some interesting applications that are of general utility in the analysis of nonstationary AM-FM signals.

\section{A. Square-Law Device}

An important nonlinear device that can be easily modeled using the results of the preceding section is the square-law operator. Thus, suppose that the squared magnitude of the linearly filtered signal (4) is computed, as depicted in Fig. 5. By Lemma 2, we have that

$$
|d(m)|^{2} \approx a^{2}(m) \cdot\left|G\left[e^{j \dot{\phi}(m)}\right]\right|^{2}
$$

for sufficiently smooth $a(m)$ and $\dot{\phi}(m)$ and localized $g(m)$ with the error falling below

$$
\begin{aligned}
& 2 a_{\max } \sum_{\substack{p \in Z \\
p \neq 0}} \bar{g}|g(p)| \int_{m-p^{+}}^{m-p^{-}}|\dot{a}(v)|+a_{\max }|p||\ddot{\phi}(v)| d v \\
& \leq 2 a_{\max } \sqrt{\gamma}\left[\bar{g} \Delta_{1}(g)\|a\|_{\mathcal{D}^{1}}+a_{\max } \bar{g} \Delta_{2}(g)\|\phi\|_{\mathcal{D}^{2}}\right] .
\end{aligned}
$$

Likewise, for a real signal $s(m)$ given by (12), we have the approximation

$$
[s(m)]^{2} \approx a^{2}(m) \cdot\left|H\left[e^{j \dot{\phi}(m)}\right]\right|^{2} \cdot \cos ^{2}\{\phi(m)+\angle H[\dot{\phi}(m)]\} .
$$

From Corollary 2, the error in (25) falls below the bounds (23) and (24).

\section{B. Teager-Kaiser Operator}

We will now apply these results to obtain limits on the interesting discrete nonlinear operators

$$
\Psi\{s(m)\}=[s(m)]^{2}-s(m+1) s(m-1)
$$

for filtered real signals and for filtered complex signals (where superscript ' $*$ ' denotes conjugation)

$$
\Phi\{d(m)\}=|d(m)|^{2}-\operatorname{Re}\left\{[d(m+1)]^{*} d(m-1)\right\} .
$$

It should be noted that we are applying the operators $\Psi\{\cdot\}$ and $\Phi\{\cdot\}$ to filtered versions of the signals $c(m)$ and $r(m)$; from the more general result obtained, new bounds on the errors of the unfiltered approximations will also be obtained.

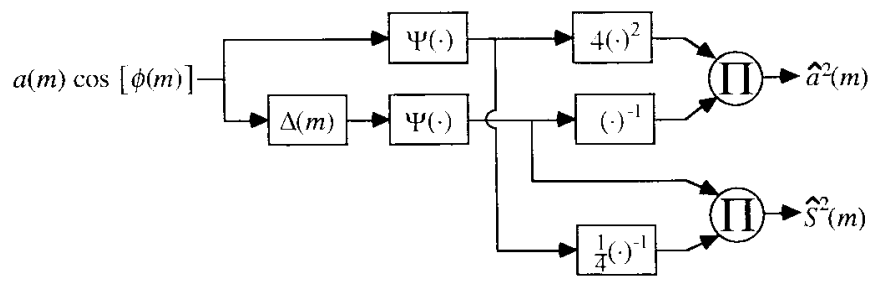

Fig. 6. Basic discrete-time energy separation algorithm. Here $\Delta(\cdot)$ denotes the two-point centered difference operation: $\Delta[r(m)]=r(m+1)-r(m-1)$.

The real-valued Teager-Kaiser operator $\Psi\{\cdot\}$, which was first proposed by Teager [9] and subsequently investigated by Kaiser and others [4]-[10] has been shown to be effective for AM and FM information demodulation in speech signals [8], [9], and, more recently, digital image analysis [13]. The discrete-time operator $\Psi\{\cdot\}$ has a continuous analog $\Psi_{c}\{\cdot\}$ defined on continuous signals $x(t)$ by

$$
\Psi_{c}\{x(t)\}=[\dot{x}(t)]^{2}-x(t) \ddot{x}(t) .
$$

Assuming a unit sampling period, we may exactly derive the discrete-time operator from the continuous operator by replacing $x(t)$ with $x(n),[\dot{x}(t)]^{2}$ with $[x(n+1)-x(n)][x(n)-$ $x(n-1)]$ (using a product of a forward and a backward difference yields a centered estimate of the squared derivative), and finally, replacing $\ddot{x}(t)$ with $x(n+1)-2 x(n)+x(n-1)$ (using a forward difference followed by a backward difference yields a centered estimate of the twice derivative).

Returning to discrete signals only, for unfiltered real AMFM signals of the form (2)

$$
\Psi\{r(m)\} \approx \hat{\Psi}\{r(m)\}=a^{2}(m)[\sin \dot{\phi}(m)]^{2}
$$

with negligible error under bandlimiting conditions on the modulating functions [6]-[8], [11]. Similarly

$$
\Psi\{r(m+1)-r(m-1)\} \approx 4 a^{2}(t)[\sin \dot{\phi}(m)]^{4}
$$

which suggests the energy separation algorithm (ESA) [6]-[8], [11]:

$$
\begin{aligned}
& \hat{a}^{2}(m)=4 \Psi^{2}\{r(m)\} / \Psi\{r(m+1)-r(m-1)\} \\
& \hat{S}^{2}(m)=\Psi\{r(m+1)-r(m-1)\} / 4 \Psi\{r(m)\}
\end{aligned}
$$

for estimating the squared amplitude envelope $a^{2}(m)$ and of the squared sine of instantaneous frequency $S^{2}(m)=$ $[\sin \dot{\phi}(m)]^{2}$, respectively. The ESA is diagrammed in Fig. 6 .

For the complex operator, we have, for the unfiltered case

$$
\begin{aligned}
\Phi\{c(m)\} \approx \hat{\Phi}\{c(m)\} & =2 a^{2}(m)[\sin \dot{\phi}(m)]^{2} \\
\Phi\{c(m+1)-c(m-1)\} & \approx 8 a^{2}(t)[\sin \dot{\phi}(m)]^{4}
\end{aligned}
$$

which suggests the ESA:

$$
\begin{aligned}
& \hat{a}^{2}(m)=2 \Phi^{2}\{c(m)\} / \Phi\{c(m+1)-c(m-1)\} \\
& \hat{S}^{2}(m)=\Phi\{c(m+1)-c(m-1)\} / 4 \Phi\{c(m)\} .
\end{aligned}
$$

The approximations (28) and (29), and by trivial extension (30) and (31), have been shown to hold quite well under assumptions on the bandwidths of the amplitude modulation functions and the instantaneous frequencies. Detailed analyses are presented in [6]-[8] and [11], which show that the relative 


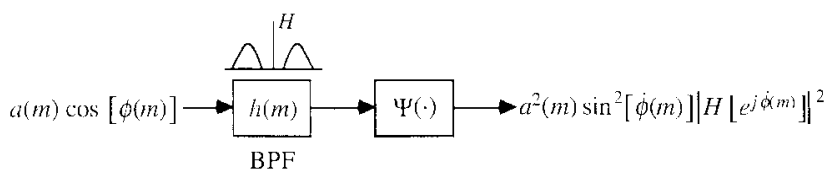

Fig. 7. Diagram of basic single-band discrete-time energy operator and approximate response to a real AM-FM signal.

error is quite small for realistic signals in speech and other communications applications, provided that the AM and FM functions obey a certain bandlimitedness constraint. As a byproduct of the ensuing analysis, we provide additional useful bounds that are bandwidth-independent.

In practical applications, it is generally necessary that the energy operators $\Phi$ and $\Psi$ be preceded by a linear filter (usually bandpass) in order to counteract the effects of noise [11] or cross-component interference. For continuous signals, such a combined operator was referred to as a single-band energy operator in [11]. For the approximations of the energies of filtered signals, we have

$$
\begin{aligned}
& \hat{\Phi}\{d(m)\}=2 a^{2}(m)[\sin \dot{\phi}(m)]^{2}\left|G\left[e^{j \dot{\phi}(m)}\right]\right|^{2} \\
& \hat{\Psi}\{s(m)\}=a^{2}(m)[\sin \dot{\phi}(m)]^{2}\left|H\left[e^{j \dot{\phi}(m)}\right]\right|^{2}
\end{aligned}
$$

as depicted in Fig. 7 for real-valued signals.

Theorem 2, which is the main result for energy operators of the type considered here, gives satisfactory bounds on the errors incurred by the approximations (32) and (33). In Theorem 2, the following shorthand notation is used: $g_{+}(m)=$ $g(m+1)$ and $g_{-}(m)=g(m-1)$ for $m \in Z$.

Theorem 2: Let

$$
\varepsilon_{\Phi}(m)=|\Phi\{d(m)\}-\hat{\Phi}\{d(m)\}|
$$

where $\Phi\{d(m)\}, \hat{\Phi}\{d(m)\}$ are given by (27) and (32). Then

$$
\begin{aligned}
\varepsilon_{\Phi}(m) \leq & a_{\max } \bar{g} \sum_{\substack{p \in Z \\
p \neq 0}}\left[\left|g_{+}(p)\right|+2|g(p)|+\left|g_{-}(p)\right|\right] \\
& \cdot \int_{m-p^{+}}^{m-p^{-}}|\dot{a}(v)|+a_{\max }|p||\ddot{\phi}(v)| d v \\
\leq & a_{\max } \sqrt{\gamma} \bar{g}\left\{\left[\Delta_{1}\left(g_{+}\right)+2 \Delta_{1}(g)+\Delta_{1}(g-)\right]\|a\|_{\mathcal{D}^{1}}\right. \\
& \left.+a_{\max }\left[\Delta_{2}\left(g_{+}\right)+2 \Delta_{2}(g)+\Delta_{2}\left(g_{-}\right)\right]\|\phi\| \|_{\mathcal{D}^{2}}\right\} .
\end{aligned}
$$

Proof: See the Appendix.

The analogous result for operator $\Psi$ and real input now follows easily and so is stated without proof.

Corollary 3: Let

$$
\varepsilon_{\Psi}(m)=|\Psi\{s(m)\}-\hat{\Psi}\{s(m)\}|
$$

where $\Psi\{s(m)\}, \hat{\Psi}\{s(m)\}$ are given by (26) and (33). Then

$$
\begin{aligned}
\varepsilon_{\Psi}(m) \leq & a_{\max } \bar{h} \sum_{\substack{p \in Z \\
p \neq 0}}\left[\left|h_{+}(p)\right|+2|h(p)|+\left|h_{-}(p)\right|\right] \\
& \cdot \int_{m-p^{+}}^{m-p^{-}}|\dot{a}(v)|+a_{\max }|p||\ddot{\phi}(v)| d v \\
\leq & a_{\max } \sqrt{\gamma} \bar{h}\left\{\left[\Delta_{1}\left(h_{+}\right)+2 \Delta_{1}(h)+\Delta_{1}\left(h_{-}\right)\right]\|a\|_{\mathcal{D}^{1}}\right. \\
& \left.+a_{\max }\left[\Delta_{2}\left(h_{+}\right)+2 \Delta_{2}(h)+\Delta_{2}\left(h_{-}\right)\right]\|\phi\|_{\mathcal{D}^{2}}\right\} .
\end{aligned}
$$

Proof: The proof has been omitted for brevity.

We now reinvestigate the operators and their approximations (28)-(31). By applying Theorem 2 and Corollary 3, we will be able to derive some approximation bounds that are complementary to those given in [7] for the real-valued operator.

Example 2: Here, we give approximation bounds on the operator outputs for unfiltered input signals; take the filter impulse responses to be the Kronecker function: $g(m)=$ $h(m)=\delta(m)=1$ for $m=0$, and $\delta(m)=0, m \neq 0$. Here, $\left|G\left(e^{j \omega}\right)\right|=\left|H\left(e^{j \omega}\right)\right|=1$ for all $\omega \in[0,2 \pi]$. Thus, the approximations are in perfect agreement with (28) and (30):

$$
\begin{aligned}
& \Phi\{c(m)\} \approx \hat{\Phi}\{c(m)\}=2 a^{2}(m)[\sin \dot{\phi}(m)]^{2} \\
& \Psi\{r(m)\} \approx \hat{\Psi}\{r(m)\}=a^{2}(m)[\sin \dot{\phi}(m)]^{2} .
\end{aligned}
$$

Since $\bar{\delta}=1, \Delta_{k}(\delta)=0$, and $\Delta_{k}\left(\delta_{+}\right)=\Delta_{k}\left(\delta_{-}\right)=1$ for $k=1,2$, then by Theorem 2 and Corollary 3 , both approximations (34) and (35) have errors that are bounded above according to

$$
\begin{aligned}
\varepsilon_{\Phi}(m), \varepsilon_{\Psi}(m) & \leq a_{\max } \int_{m-1}^{m+1}|\dot{a}(v)|+a_{\max }|\ddot{\phi}(v)| d v \\
& \leq 2 a_{\max } \sqrt{\gamma}\left\{|| a\left\|_{\mathcal{D}^{1}}+a_{\max }|| \phi\right\|_{\mathcal{D}^{2}}\right\}
\end{aligned}
$$

In the interesting case of a real-valued chirp signal $r(m)=$ $A \cos \left(B m^{2}+C m+D\right)$, the approximate response of $\Psi\{\cdot\}$ is then

$$
\hat{\Psi}\{r(m)\}=A^{2} \sin ^{2}[(2 B m+C)]
$$

with an error that falls below $4 A^{2}|B|$. A nearly identical result holds for the complex chirp signal. It should be noted that this is the first available result that usefully bounds the error of the frequently used approximations (34) and (35) for a chirp signal.

Finally, note that the approximations (34) and (35) are always nonnegative, in accordance with an interpretation of $\Phi\{\cdot\}$ and $\Psi\{\cdot\}$ as energy. However, as shown in [15], where necessary and sufficient conditions were given for positivity of $\Psi\{\cdot\}$, there is no guarantee of this in practice.

Example 3: Next, we give approximation bounds for the difference operator outputs (29) and (31); for the complex operator, take the filter impulse responses to be

$$
g(m)=h(m)=[\delta(m+1)-\delta(m-1)] / \sqrt{2} .
$$

Then

$$
\left|G\left(e^{j \omega}\right)\right|=\left|H\left(e^{j \omega}\right)\right|=\sqrt{2}|\sin \omega| \text { for all } \omega \in[0,2 \pi] .
$$

Thus, the approximations are

$$
\begin{aligned}
\Phi\{c(m+1)-c(m-1)\} & \approx \hat{\Phi}\{c(m+1)-c(m-1)\} \\
& =4 a^{2}(m)[\sin \dot{\phi}(m)]^{4} \\
\Psi\{r(m+1)-r(m-1)\} & \approx \hat{\Psi}\{r(m+1)-r(m-1)\} \\
& =2 a^{2}(m)[\sin \dot{\phi}(m)]^{4} .
\end{aligned}
$$

Again, using Theorem 2 and Corollary 3, both approximations (36) and (37) are bounded above by

$$
12 a_{\max } \sqrt{\gamma}\left\{\|a\|_{\mathcal{D}^{1}}+3 a_{\max }\|\phi\|_{\mathcal{D}^{2}}\right\} .
$$




\section{ApPlication to a Discrete Multiband AM-FM DEMODULATION SCHEME}

As a more extensive application of the theory developed in the preceding, we shall now explore a simple multiband demodulation algorithm; as a byproduct, an optimal class of filters is derived using a discrete uncertainty principle criterion recently developed by Doroslovacki et al. [16].

\section{A. Multiband Demodulation Algorithm}

Suppose that a complex-valued AM-FM signal $c(m)$ as in (1) is passed through a set of unit-energy bandpass filters $h_{n}(m) ; n=1, \cdots, N$, yielding outputs $d_{n}(m)=h_{n}(m) *$ $c(m)$. Although the sampling of the frequency plane with the filter bank might be designed to accomplish a complete, orthonormal (wavelet) decomposition of the input signal, this need not be assumed for the AM-FM demodulation process to be analyzed. Indeed, we will not even explore the frequency tessellation here; instead, the filters $h_{n}(m)$ are simply assumed to sample the spectrum $[0, \pi]$ sufficiently densely that a large response is assured at each $m$; see, e.g., [1]-[3], [11], and [12].

For each instant $m_{0}$, the normalized response (defined over all $m$ )

$$
y_{0}(m)=\frac{d_{n}(m)}{H_{n, \max }}
$$

corresponding to the response having the maximum normalized amplitude at $m_{0}$

$$
n=\arg \max _{1 \leq i \leq N} \frac{\left|d_{i}\left(m_{0}\right)\right|}{H_{i, \max }}
$$

where

$$
H_{n, \max }=\sup _{\omega}\left|H_{n}\left(e^{j \omega}\right)\right| .
$$

For simplicity, denote the channel that maximizes (38) at time $m_{0}$ by $h_{0}(m)$; in addition, assume $H_{n, \max }=1$ for $1 \leq n \leq N$. At moment $m_{0}, h_{0}(m)$ captures a relatively large percentage of the local AM-FM signal energy. To estimate this information, first define

$$
z(m)=\left[y_{0}(m+K)+y_{0}(m-K)\right] / 2
$$

which is equivalent to filtering with

$$
g(m)=\left[h_{0}(m+K)+h_{0}(m-K)\right] / 2 .
$$

A simple AM-FM demodulation algorithm is then

$$
\hat{\dot{\phi}}(m)=\frac{1}{K} \cdot \operatorname{Cos}^{-1}\left[\frac{z(m)}{y_{0}(m)}\right] \approx \dot{\phi}(m)
$$

and

$$
\hat{a}(m)=\left|\frac{y_{0}(m)}{H_{0}\left(e^{j \hat{\phi}(m)}\right)}\right| \approx a(m) .
$$

By using quantities that incorporate the filter response (38) having the maximum amplitude, one ensures that in practice, the AM-FM energy that is captured is maximized relative to any ambient noise or other subcomponents. In (39)-(42), a two-point difference, rather than average of filter outputs can

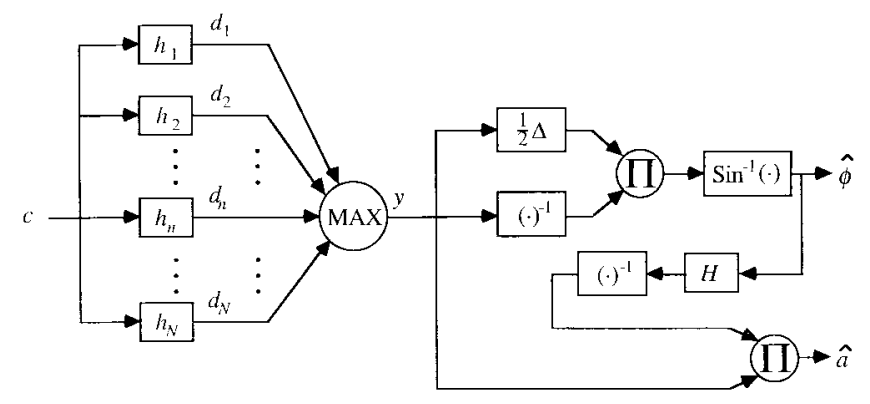

Fig. 8. Flow diagram depicting multiband filtering and energy separation of an AM-FM signal. For this system the operator $\Delta(\cdot)$ is defined as in Fig. 6.

also be used, as is depicted in the flow diagram in Fig. 8 using the ordinary centered difference $(K=1)$. However, calculating $\hat{\phi}(m)$ in (41) then requires finding an unambiguous interpretation for $\operatorname{Sin}^{-1}$ on $[0, \pi]$; in addition, (39) has the advantage of being resistant to high-frequency noise, which can be a problem with operators of this type [11].

\section{B. Error Analysis and Optimal Filter Selection}

Approximations (41) are (42) are supported as follows. By (5) and Lemma $1, z(m) \approx \hat{z}(m)$, where

$$
\hat{z}(m)=c(m) \cos [K \dot{\phi}(m)] H_{0}\left[e^{j \dot{\phi}(m)}\right]
$$

with an error bounded above by

$$
\begin{aligned}
e_{z}(m)= & |z(m)-\hat{z}(m)| \leq \sqrt{\gamma}\left[\Delta_{1}(g) \cdot\|a\|_{\mathcal{D}^{1}}\right. \\
& \left.+a_{\max } \Delta_{2}(g)\|\phi\|_{\mathcal{D}^{2}}\right] .
\end{aligned}
$$

Here, we are only making use of the bounds that separate the (nonnormalized) filter durations from the global smoothness measures since they will be used directly in a filter design procedure.

Assume that the maximizing filter in (38) may be written $h(m)=w(m) \exp \left(j \omega_{c} m\right)$. Then, by an application of Minkowski's inequality, we have that

$$
\begin{aligned}
\Delta_{k}(g) \leq & \Delta_{k}\{[w(m+K)+w(m-K)] / 2\} \\
& +\Delta_{k}\{[w(m+K)-w(m-K)] / 2\} \\
= & \partial_{k, K}(w)+\varepsilon_{k, K}(w)
\end{aligned}
$$

where

$$
\partial_{k, K}(w)=\sqrt{\frac{1}{2 \pi} \int_{-\pi}^{\pi}\left|\frac{d^{k}}{d \omega^{k}} \cos (K \omega) W\left(e^{j \omega}\right)\right|^{2} d \omega}
$$

and

$$
\varepsilon_{k, K}(w)=\sqrt{\frac{1}{2 \pi} \int_{-\pi}^{\pi} \mid \frac{d^{k}}{d \omega^{k}} \sin (K \omega) W\left(\left.e^{j \omega)}\right|^{2} d \omega\right.}
$$

with $w(m) \leftrightarrow W\left(e^{j \omega}\right)$. Selecting $w(m)$ so that $\partial_{k, K}(w)$ is small is a localization criterion that partly controls the accuracy of (43). In fact, $\partial_{k, K}(w)=0$ for the solutions of the difference equation

$$
w(m+K)+w(m-K)=C \cdot \delta(m)
$$

which are given by $W\left(e^{j \omega}\right) \propto \sec (K \omega)$, which is not useful by itself since it is not of finite energy. Forcing $\varepsilon_{k, K}(w)$ 


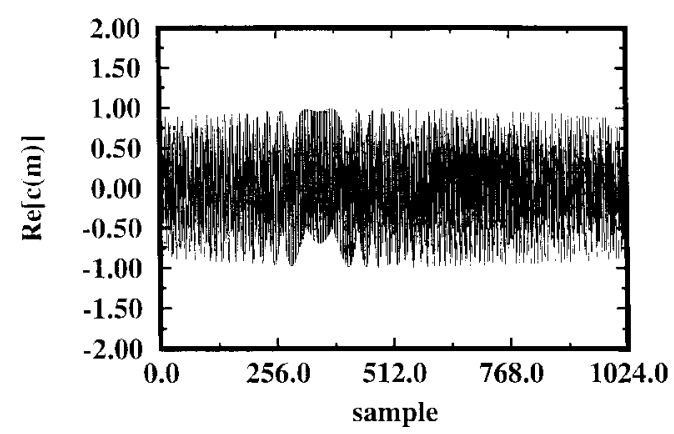

(a)

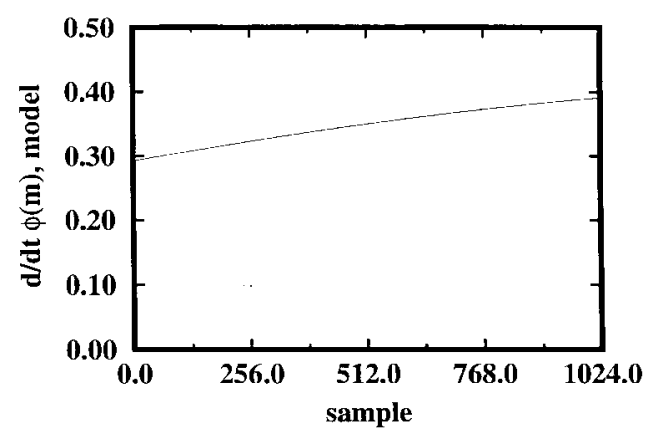

(c)

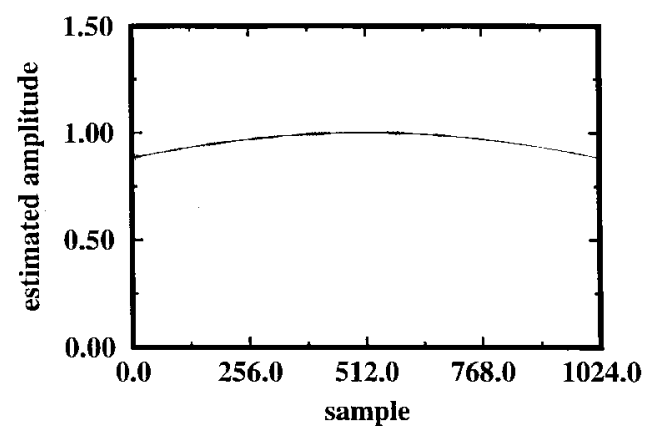

(e)

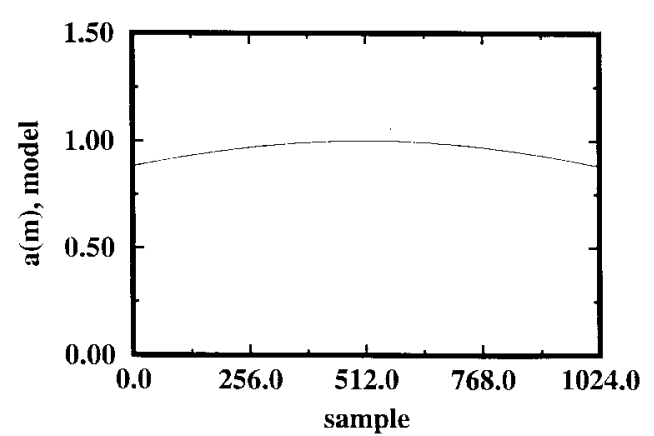

(b)

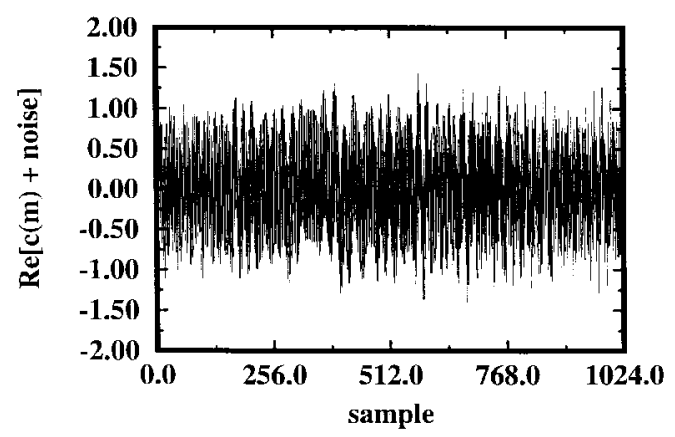

(d)

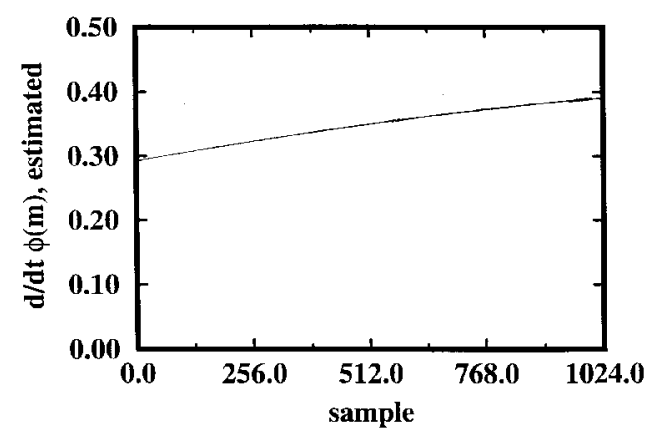

(f)

Fig. 9. Multiband demodulation of a noisy AM-FM signal. (a) Real part of original AM-FM signal. (b) Exact AM function $a(m)$. (c) Exact FM function $\dot{\phi}(m)$. (d) Signal plus noise, SNR $=2$. (e) $\hat{a}(m)$ is the AM ESA approximation. (f) $\dot{\phi}(m)$ is the FM ESA approximation.

small is a smoothness criterion that induces small variation in $\hat{a}(m)$ and $\dot{\phi}(m)$. This is desirable, since estimating a signal's AM-FM components is generally a difficult, ill-posed problem [11]. In a related 2-D continuous-domain image processing problem [3], the estimated AM-FM functions were constrained by forcing $\|a\|_{\mathcal{D}^{1}}$ and $\|\phi\|_{\mathcal{D}^{2}}$ small.

Since the AM-FM estimates are computed rather directly from the filter outputs, a simple approach to force smoothness at the output is to minimize

$$
\begin{aligned}
& \sqrt{\frac{1}{4} \sum_{m \in Z}|h(m+K)-h(m-K)|^{2}} \\
& \quad \leq \Delta_{0}\left\{\frac{1}{2}[w(m+K)-w(m-K)]\right\} \\
& \quad+\Delta\left\{\frac{1}{2}[w(m+K)+w(m-K)]\right\} \\
& =\varepsilon_{0, K}(w)+\partial_{0, K}(w) .
\end{aligned}
$$

In view of the preceding, it is of sufficient interest to minimize $\partial_{1, K}(w)$ (hence localized $g$ ) and $\varepsilon_{0, K}(w)$ (smooth estimates). Simultaneously forcing $\partial_{1, K}(w)$ and $\varepsilon_{0, K}(w)$ small are conflicting goals as expressed by the Doroslovacki-Fan-Djuric uncertainty relation [16], which states that for $K=1 / 2$

$$
\partial_{1, K}(w) \cdot \varepsilon_{0, K}(w) \geq 1 / 4 .
$$

The filters that uniquely achieve the lower bound in (44) have the form $(q>-1 / 2)[16]$ :

$$
W\left(e^{j \omega}\right)=B(q)[2 \cos (\omega / 2)]^{q}
$$

where

$$
B(q)=\frac{\Gamma(q+1)}{\sqrt{\Gamma(2 q+1)}}
$$

yields unit energy $[\Gamma(\cdot)=$ gamma function]. The optimal filters (45) maintain localized low-frequency energy while simultaneously de-emphasizing high-frequency energy. These filters approach a Gaussian characteristic as $q \rightarrow \infty$ [16]; hence, the optimal channel filters are of the form

$$
H\left(e^{j \omega}\right)=B(q)\left\{2 \cos \left[\left(\omega-\omega_{c}\right) / 2\right]\right\}^{q}
$$


At this time, the relative merits of these filters as a function of $q$ remains an open question, other than the low uncertainty property shared by all the members of the class. However, for large $q$, these functions resemble Gabor functions [16], which is in agreement with continuous optimization formulations developed in [1]-[3], [11], and [12]. Indeed, as $q \rightarrow \infty, H\left(e^{j \omega}\right)$ has the form of a discrete-time Gabor function.

\section{Numerical Results}

The multiband demodulation system described in the preceding was implemented using five one-octave Gabor filters spaced at one-octave separations and sampling the frequency domain at center frequencies $\omega_{g}=$ $3 \pi / 64,3 \pi / 32,3 \pi / 16,3 \pi / 8$, and $3 \pi / 4$ rads (filters $1-5$, respectively). This tessellation results in filters that intersect exactly at half-peak of frequency responses.

An AM-FM input signal of the form (14)-(16) was applied to the demodulation system. The signal parameters selected were $\Delta \omega=0.2 \pi$ rads, $\sigma_{a}=1024, \sigma_{\phi}=4096$. The (real part of) the signal is plotted in Fig. 9(a). The exact AM-FM functions $a(m)$ and $\dot{\phi}(m)$ are shown in Fig. 9(b) and (c), respectively. Pseudo-random, i.i.d. zero-mean noise samples $v(m)$ from a uniform distribution were then generated, passed through filter 2 (the one-octave Gabor filter at center frequency $3 \pi / 32$ ) to create a bandpass noise signal lying in a different band than most of the signal AM-FM energy, and, finally, added to the signal. The (real part of) the noisy signal is depicted in Fig. 9(d). The SNR of the signal-plus-noise was selected to be $|c|_{\max } / v_{\max }=2$, where $|c|_{\max }$ is the maximum signal amplitude, and $v_{\max }$ is the maximum noise amplitude. Although this input signal does not supply the same rigorous test for the system as a severe in-band noise (sharing the local spectrum with the signal), the ability of the multiband demodulation system to track the AM-FM signal in moderate noise is amply demonstrated in Fig. 9(e) and (f). While a noise analysis is beyond the scope of the present paper, we expect that superior performance can be attained for most noise situations through a denser filter sampling, as demonstrated for continuous-domain demodulation systems of this type in [11].

\section{CONCLUDING REMARKS}

In this paper, we have developed generally applicable theorems for analyzing discrete AM-FM functions and their behavior in discrete linear systems, as well as simple discrete nonlinear systems comprised of products of discrete linear subsystems. Approximations for the responses of these systems to input functions of the form (1) and (2) termed quasieigenfunction approximations (QEA's) were derived that complement the eigenfunction interpretation of sinusoids in linear systems. For nonsinusoidal signals, the approximations have errors that are bounded by Sobolev norm functionals expressing the smoothness of the AM and FM information signals and in terms of discrete energy variance functionals expressing the durations of the involved system impulse responses.
AM-FM models such as (1) and (2) that capture physically meaningful signal nonstationarities are finding increased applications, particularly in instances where local nonstationary signal structures are to be modeled in a globally consistent way. New analysis techniques, expanding on those given here, will help to exploit the power of the approach. Currently, we are studying extended models of the form [12]

$$
\begin{aligned}
& c(m)=\sum_{k} a_{k}(m) e^{j \phi_{k}(m)} \\
& r(m)=\sum_{k} a_{k}(m) \cos \left[\phi_{k}(m)\right]
\end{aligned}
$$

Generalizing the model to include the possibility of multicomponents is a natural one since signals may be composed of subsignals containing multiple evolving information sets best separately modeled as modulations. As the number of components becomes very large, the models (46) and (47) in fact may be made DFT-like. This suggests the powerful notion of AM-FM representations of signals, as opposed to models, where a relatively small number of additive AM-FM components are used to exactly represent subsignals composed of locally coherent, yet nonstationary substructures. Efficient representations that capture the essential modulation signal structures using as few components as possible are of high interest.

\section{APPENDIX}

Proofs of the main results are given here.

Proof of Theorem 1: The proof of Theorem 1 relies on isolating the amplitude and phase differences between the actual response and the approximation and then bounding these differences in terms of the variation of the AM-FM functions. The channel filter impulse response weights the local variation. First note that by Taylor's theorem with remainder [14]

$$
\phi(m-p)=\phi(m)-p \dot{\phi}(m)+Q_{\phi}(m, p)
$$

where

$$
Q_{\phi}(m, p)=p^{2} \int_{0}^{1}(1-u) \ddot{\phi}(m-u p) d u
$$

From (1), (4), and (A.1)

$$
\begin{aligned}
d(m)= & a(m) \exp [j \phi(m)] * g(m) \\
= & \sum_{p \in Z} g(p) a(m-p) \exp [j \phi(m)] \exp [-j p \dot{\phi}(m)] \\
& \cdot \exp \left[j Q_{\phi}(m, p)\right]
\end{aligned}
$$

and from (5), we have

$$
\hat{d}(m)=\sum_{p \in Z} g(p) a(m) \exp [j \phi(m)] \exp [-j p \dot{\phi}(m)] .
$$

By first applying the triangle inequality to the difference between (A.3) and (A.4), introducing the zero term $a(m-$ $p)-a(m-p)$ inside the result, and applying the inequality $|\alpha-\beta| \leq|\alpha-z|+|z-\beta|$, the following bound on $\varepsilon_{d}(m)$ 
is obtained:

$$
\begin{aligned}
\varepsilon_{d}(m) \leq & \sum_{p \in Z}|g(p)|\left|a(m-p) \exp \left[j Q_{\phi}(m, p)\right]-a(m)\right| \\
\leq & \sum_{p \in Z}|g(p)||a(m-p)-a(m)| \\
& +\sum_{p \in Z}|g(p)||a(m-p)| \\
& \cdot\left|\exp \left[j Q_{\phi}(m, p)\right]-1\right| \\
\leq & \sum_{p \in Z}|g(p)||a(m-p)-a(m)| \\
& +a_{\max } \sum_{p \in \boldsymbol{Z}}|g(p)|\left|Q_{\phi}(m, p)\right| \\
= & \varepsilon_{d, a}(m)+\varepsilon_{d, \phi}(m) .
\end{aligned}
$$

However, since

$$
|a(m-p)-a(m)|=\left|\int_{m-p}^{m} \dot{a}(v) d v\right|
$$

then

$$
\varepsilon_{d, a}(m) \leq \sum_{\substack{p \in \boldsymbol{Z} \\ p \neq 0}}|g(p)| \int_{m-p^{+}}^{m-p^{-}}|\dot{a}(v)| d v
$$

where the sum is not taken at $p=0$ in order to show the interesting independence of the bound on $g(0)$.

In addition, from (A.2)

$$
\begin{aligned}
\left|Q_{\phi}(m, p)\right| & =\left|p^{2} \int_{0}^{1}(1-u) \ddot{\phi}(m-u p) d u\right| \\
& =\left|p \int_{0}^{p}(1-v / p) \ddot{\phi}(m-v) d v\right| \\
& \leq|p| \int_{p^{-}}^{p^{+}}|\ddot{\phi}(m-v)| d v \\
& =|p| \int_{m-p^{+}}^{m-p^{-}}|\ddot{\phi}(v)| d v
\end{aligned}
$$

so that

$$
\varepsilon_{d, \phi}(m) \leq a_{\max } \sum_{\substack{p \subset \boldsymbol{Z} \\ p \neq 0}}|p||g(p)| \int_{m-p^{+}}^{m-p^{-}}|\ddot{\phi}(v)| d v .
$$

Finally, combining (A.5), (A.7), and (A.9)

$$
\varepsilon_{d}(m) \leq \sum_{\substack{p \in \boldsymbol{Z} \\ p \neq 0}}|g(p)| \int_{m-p^{+}}^{m-p^{-}}|\dot{a}(v)|+a_{\max }|p||\ddot{\phi}(v)| d v .
$$

Proof of Lemma 1: The proof extends results from the proof of Theorem 1 by separating the channel impulse response from the AM-FM variation. Note that in (A.7)

$$
\int_{m-p^{+}}^{m-p^{-}}|\dot{a}(v)| d v=|p| \int_{0}^{1}|\dot{a}(m-u p)| d u
$$

and therefore

$$
\begin{aligned}
& \varepsilon_{d, a}(m) \leq \sum_{\substack{p \in Z \\
p \neq 0}}|p||g(p)| \int_{0}^{1}|\dot{a}(m-u p)| d u \\
& \leq\left[\sum_{\substack{p \in \boldsymbol{Z} \\
p \neq 0}} p^{2}|g(p)|^{2}\right]^{1 / 2} \\
& \cdot\left\{\sum_{\substack{p \in \boldsymbol{Z} \\
p \neq 0}}\left[\int_{0}^{1}|\dot{a}(m-u p)| d u\right]^{2}\right\}^{1 / 2} \\
& =\Delta_{1}(g)\left\{\sum_{\substack{p \in \boldsymbol{Z} \\
p \neq 0}} p^{-2}\left[\int_{0}^{p}|\dot{a}(m-u)| d u\right]^{2}\right\}^{1 / 2} \\
& \leq \Delta_{1}(g) \int_{\boldsymbol{R}}|\dot{a}(u)| d u \cdot\left[\sum_{\substack{p \in \boldsymbol{Z} \\
p \neq 0}} p^{-2}\right]^{1 / 2} \\
& =\sqrt{\gamma} \Delta_{1}(g) \int_{\boldsymbol{R}}|\dot{a}(u)| d u \\
& =\sqrt{\gamma} \Delta_{1}(g) \cdot\|a\|_{\mathcal{D}^{1}}
\end{aligned}
$$

where the filter and AM functions were separated by application of the Cauchy-Schwarz inequality. This bound on $\varepsilon_{d, a}(m)$ always exceeds that in (A.7).

Similarly, in (A.8)

$$
\int_{m-p^{+}}^{m-p^{-}}|\ddot{\phi}(r)| d r=|p| \int_{0}^{1}|\ddot{\phi}(m-u p)| d u
$$

and therefore, from (A.9)

$$
\begin{aligned}
\varepsilon_{d, \phi}(m) \leq & a_{\max } \sum_{\substack{p \in \boldsymbol{Z} \\
p \neq 0}} p^{2}|g(p)| \int_{0}^{1}|\ddot{\phi}(m-u p)| d u \\
\leq & a_{\max }\left[\sum_{\substack{p \in \boldsymbol{Z} \\
p \neq 0}} p^{4}|g(p)|^{2}\right]^{1 / 2} \\
& \cdot\left\{\sum_{\substack{p \in \boldsymbol{Z} \\
p \neq 0}}\left[\int_{0}^{1}|\ddot{\phi}(m-u p)| d u\right]^{2}\right\}^{1 / 2} \\
= & a_{\max } \Delta_{2}(g)\left\{\sum_{\substack{p \in \boldsymbol{Z} \\
p \neq 0}} p^{-2}\left[\int_{0}^{p}|\ddot{\phi}(m-u)| d u\right]^{2}\right\}^{1 / 2} \\
\leq & a_{\max } \Delta_{2}(g) \int_{\boldsymbol{R}}|\ddot{\phi}(u)| d u\left[\sum_{\substack{p \in \boldsymbol{Z} \\
p \neq 0}} p^{-2}\right]^{1 / 2} \\
= & a_{\left.\max \sqrt{\gamma} \Delta_{2}(g)\|\phi\| .12\right)}
\end{aligned}
$$


The proof is completed by combining (A.5), (A.11), and (A.12)

$$
\varepsilon_{d}(m)=\varepsilon_{d} \leq \sqrt{\gamma} \Delta_{1}(g) \cdot\|a\|_{\mathcal{D}^{1}}+a_{\max } \sqrt{\gamma} \Delta_{2}(g)\|\phi\|_{\mathcal{D}^{2}}
$$

where the dependence on $m$ has been removed.

Proof of Corollary 1: We proceed by breaking the error into two terms corresponding to positive and negative exponents of frequency and then applying the results of earlier proofs to each of these terms. Introducing a bivalued variable $q \in\{-1,1\}$ to index positive and negative exponents, we have from (4), (A.1), (A.2), and Euler's identity

$$
\begin{aligned}
s(m)= & \left(\frac{1}{2}\right) \sum_{q \in\{-1,1\}} \sum_{p \in Z} h(p) a(m-p) \exp [j q \phi(m)] \\
& \cdot \exp [-j p q \dot{\phi}(m)] \exp \left[j q Q_{\phi}(m, p)\right]
\end{aligned}
$$

and from (13), we have

$$
\begin{aligned}
\hat{s}(m)= & \left(\frac{1}{2}\right) \sum_{q \in\{-1,1\}} \sum_{p \in Z} h(p) a(m) \exp [j q \phi(m)] \\
& \cdot \exp [-j p q \dot{\phi}(m)] .
\end{aligned}
$$

Following the reasoning in the proof of Theorem 1, we have that

$$
\begin{aligned}
\varepsilon_{s}(m) \leq & \left(\frac{1}{2}\right) \sum_{q \in\{-1,1\}} \sum_{p \in Z}|h(p)| \mid a(m-p) \\
& \cdot \exp \left[j q Q_{\phi}(m, p)\right]-a(m) \mid \\
\leq & \left(\frac{1}{2}\right) \sum_{q \in\{-1,1\}} \sum_{p \in Z}|h(p)| a(m-p) \| \\
& \cdot\left|\exp \left[j q Q_{\phi}(m, p)\right]-1\right| \\
& +\left(\frac{1}{2}\right) \sum_{q \in\{-1,1\}} \sum_{p \in Z}|h(p)||a(m-p)-a(m)| \\
\leq & a_{\max }\left(\frac{1}{2}\right) \sum_{q \in\{-1,1\}}|h(p)|\left|q Q_{\phi}(m, p)\right|+ \\
& \left(\frac{1}{2}\right) \sum_{q \in\{-1,1\}} \sum_{p \in Z}|h(p) \| a(m-p)-a(m)| \\
= & \left(\frac{1}{2}\right) \cdot 2 \cdot \varepsilon_{s, \phi}(m)+\left(\frac{1}{2}\right) \cdot 2 \cdot \varepsilon_{s, a}(m) \\
= & \varepsilon_{s, \phi}(m)+\varepsilon_{s, a}(m) .
\end{aligned}
$$

The proof then follows easily from (A.7), (A.9), (A.11) and (A.12).

Proof of Lemma 2: Results from previous proofs can also be applied here. We have from (1), (4), (A.1), and (17)

$$
\begin{aligned}
b(m)= & \sum_{p \in Z} \sum_{q \in Z} g_{1}(p)\left[g_{2}(q)\right]^{*} a(m-p) a(m-q) \\
& \cdot \exp [-j(p-q) \dot{\phi}(m)] \\
& \cdot \exp \left\{j\left[Q_{\phi}(m, p)-Q_{\phi}(m, q)\right]\right\}
\end{aligned}
$$

and from (6) and (18)

$$
\hat{b}(m)=\sum_{p \in Z} \sum_{q \in Z} g_{1}(p)\left[g_{2}(q)\right]^{*} a^{2}(m) \exp [-j(p-q) \dot{\phi}(m)] \text {. }
$$

Hence

$$
\begin{aligned}
e_{b}(m)= & \sum_{p \in Z} \sum_{q \in Z}\left|g_{1}(p)\right| g_{2}(q)|| a(m-p) a(m-q) \\
& \cdot \exp \left\{j\left[Q_{\phi}(m, p)-Q_{\phi}(m, q)\right]\right\}-a^{2}(m) \mid \\
\leq & \sum_{p \in Z} \sum_{q \in Z}\left|g_{1}(p)\right|\left|g_{2}(q)\right| \mid a(m-p) a(m-q) \\
& -a^{2}(m)\left|+a_{\max }^{2} \sum_{p \in Z} \sum_{q \in Z}\right| g_{1}(p)|| g_{2}(q) \mid \\
& \cdot\left\{\left|Q_{\phi}(m, p)\right|+\left|Q_{\phi}(m, q)\right|\right\} \\
\leq & a_{\max } \cdot \bar{g}_{2} \sum_{p \in Z}\left|g_{1}(p)\right||a(m-p)-a(m)| \\
& +a_{\max } \cdot \bar{g}_{1} \sum_{q \in Z}\left|g_{2}(q)\right||a(m-q)-a(m)| \\
& +a_{\max }^{2} \cdot \bar{g}_{2} \sum_{p \in Z}\left|g_{1}(p)\right|\left|Q_{\phi}(m, p)\right| \\
& \cdot+a_{\max }^{2} \bar{g}_{1} \sum_{q \in Z}\left|g_{2}(q)\right|\left|Q_{\phi}(m, q)\right| .
\end{aligned}
$$

The first two summations following the final inequality in (A.18) can be identically bounded as in (A.7), whereas the latter two summations can be identically bounded as in (A.9). Combining these yields the desired result (19). Likewise, applying (A.11) and (A.12) to (A.18) yields (20).

Proof of Theorem 2: By Lemma 2

$$
\begin{aligned}
& \|\left. d(m)\right|^{2}-a^{2}(m)\left|G\left[e^{j \dot{\phi}(m)}\right]\right|^{2} \\
& \leq 2 a_{\max } \bar{g} \sum_{\substack{p \in Z \\
p \neq 0}}|g(p)| \int_{m-p^{+}}^{m-p^{-}}|\dot{a}(v)| \\
& \quad+a_{\max }|p \| \ddot{\phi}(v)| d v \\
& \leq 2 a_{\max } \sqrt{\gamma} \bar{g}\left[\Delta_{1}(g)\|a\|_{\mathcal{D}^{1}}+a_{\max } \Delta_{2}(g)\|\phi\|_{\mathcal{D}^{2}}\right] .
\end{aligned}
$$

Note that $d(m \pm 1)=c(m) * g(m \pm 1)$ and recall (27) and (32). Then, also by Lemma 2

$$
\begin{aligned}
\mid \operatorname{Re}\left\{[d(m+1)]^{*} d(m-1)\right\}-2 a^{2}(m)[\sin \dot{\phi}(m)]^{2} \\
\cdot\left|G\left[e^{j \dot{\phi}(m)}\right]\right|^{2} \mid \\
\leq a_{\max } \bar{g} \sum_{\substack{p \in Z \\
p \neq 0}}\left[\left|g_{+}(p)\right|+\left|g_{-}(p)\right|\right] \int_{m-p^{+}}^{m-p^{-}}|\dot{a}(v)| \\
\quad+a_{\max } \| \ddot{\phi}(v) \mid d v \\
\leq a_{\max } \sqrt{\gamma} \bar{g}\left\{\left[\Delta_{1}\left(g_{+}\right)+\Delta_{1}\left(g_{-}\right)\right]\|a\|_{\mathcal{D}^{1}}\right. \\
\left.\quad+a_{\max }\left[\Delta_{2}\left(g_{+}\right)+\Delta_{2}\left(g_{-}\right)\right]\|\phi\|_{\mathcal{D}^{2}}\right\}
\end{aligned}
$$

where for $m \in Z$, we denote $g_{ \pm}(m)=g(m \pm 1)$. Combining (A.19) and (A.21) completes the proof.

\section{ACKNOWLEDGMENT}

The authors would like to thank Reviewer 1 and Reviewer 2, who both supplied comments that helped to clarify the paper by quite a bit. 


\section{REFERENCES}

[1] A. C. Bovik, M. Clark, and W. S. Geisler, "Multichannel texture analysis using localized spatial filters," IEEE Trans. Pattern Anal. Machine Intell., vol. 12, pp. 55-73, Jan. 1990.

[2] A. C. Bovik, "Analysis of multichannel narrowband filters for image texture segmentation," IEEE Trans. Signal Processing, vol. 39, pp. 2025-2043, Sept. 1991.

[3] A. C. Bovik, N. Gopal, T. Emmoth, and A. Restrepo, "Localized measurement of emergent image frequencies by Gabor wavelets," IEEE Trans. Inform. Theory, Special Issue on Wavelet Transforms and Multiresolution Signal Analysis, vol. 38, pp. 691-712, Mar. 1992.

[4] P. Maragos, A.C. Bovik and T.F. Quatieri, "A multidimensional energy operator for image processing," in Proc. SPIE Symp. Visual Commun. Image Processing, Boston, MA, Nov. 1992.

[5] J. F. Kaiser, "On a simple algorithm to calculate the "energy' of a signal," in Proc. IEEE Int. Conf. Acoust., Speech, Signal Processing, Albuquerque, NM, Apr. 1990.

[6] P. Maragos, T. F. Quatieri, and J. F. Kaiser, "Speech nonlinearities, modulations, and energy operators," in Proc. IEEE Int. Conf. Acoust., Speech, Signal Processing, Toronto, Ont., Canada, May 1991.

[7] _ "On amplitude and frequency demodulation using energy operators," IEEE Trans. Signal Processing, vol. 41, pp. 1532-1550, Apr. 1993.

[8] _ "Energy separation in signal modulations with applications to speech analysis," IEEE Trans. Signal Processing, vol. 41, pp. 3024-3051, Oct. 1993.

[9] H. M. Teager and S. M. Teager, "Evidence for nonlinear speech production mechanisms in the vocal tract," in Proc. NATO Adv. Study Inst. Speech Prod. Speech Model., Bonas, France, July 1989, pp. 241-261.

[10] J. F. Kaiser, "On Teager's energy algorithm and its generalization to continuous signals," in Proc. IEEE DSP Workshop., New Paltz, NY, Sept. 1990.

[11] A. C. Bovik, P. Maragos and T. F. Quatieri, "AM-FM energy detection and separation in noise using multiband energy operators," IEEE Trans. Signal Processing, Special Issue on Wavelets and Signal Processing, vol. 41, pp. 3245-3265, Dec. 1993

[12] J. P. Havlicek and A. C. Bovik, "Multi-component AM-FM image models and wavelet-based demodulation with component tracking," in Proc. IEEE Int. Conf. Image Processing, Austin, TX, Nov. 1994.

[13] P. Maragos and A. C. Bovik, "Image amplitude and frequency demodulation using multidimensional energy separation," J. Opt. Soc. Amer., 1995.

[14] J. E. Marsden and A. J. Tromba, Vector Calculus. San Francisco, CA: W. H. Freeman, 1981

[15] A. C. Bovik and P. Maragos, "Conditions for positivity of an energy operator," IEEE Trans. Signal Processing, vol. 42, pp. 469-471, Feb. 1994.

[16] M. Doroslovacki, H. Fan, and P. M. Djuric, "Time-frequency localization for sequences," Proc. IEEE Int. Symp. Time-Freq. Time-Scale Anal., Victoria, B.C., Oct. 1992.

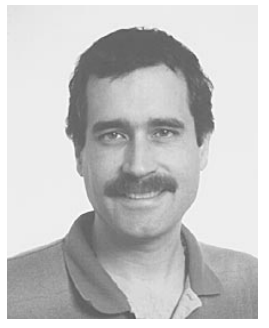

Alan C. Bovik (F'94) received the Ph.D. degree in electrical and computer engineering from the University of Illinois, Urbana-Champaign, in 1984.

$\mathrm{He}$ is currently the General Dynamics Endowed Fellow and Professor in the Department of Electrical and Computer Engineering at the University of Texas at Austin, where he is the Associate Director of the Center for Vision and Image Sciences. During the Spring of 1992, he held a visiting position in the Division of Applied Sciences, Harvard University, Cambridge, MA. His current research interests include image processing, computer vision, wavelets, 3-D microscopy, and computational aspects of biological visual perception. He has published over 200 technical articles in these areas and holds U.S. patents for the image and video compression algorithms VPIC and VPISC.

Dr. Bovik has served in numerous professional society activities, including Board of Governors, IEEE Signal Processing Society, 1996-present; Editorin-Chief, IEEE Transactions on Image Processing, 1996-present; and Founding General Chairman, First IEEE International Conference on Image Processing, Austin, TX, November 1994. He is a two-time Honorable Mention winner of the International Pattern Recognition Society Award for Outstanding Contribution (1988 and 1993). He is a registered Professional Engineer in the State of Texas and is a frequent consultant to industry and academic institutions.

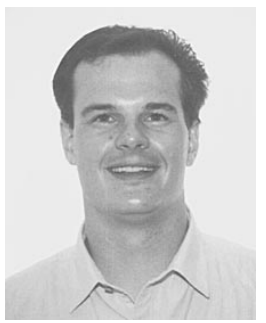

Joseph P. Havlicek (S'90-M'96) was born in West Lafayette, IN, on April 7, 1964. He received the B.S. degree in 1986 and the M.S. degree in 1988 from Virginia Polytechnic Institute and State University, Blacksburg, and the Ph.D. degree in 1996 from the University of Texas at Austin, all in electrical engineering.

From 1984 to 1987, he was with Management Systems Laboratories, Blacksburg, VA, as a software engineer. From 1987 to 1989 , he was affiliated with SFA, Inc., Landover, MD, and from 1987 to 1996, he was with the Naval Research Laboratory, Washington, DC, where his work included high-performance signal and image processing. In 1993, he was a programmer-analyst with Ralph Kirkley Associates, Austin, TX, working on-site in the multimedia division of IBM, Austin. Since January 1997, he has been an Assistant Professor in the School of Electrical and Computer Engineering at the University of Oklahoma, Norman. His research interests include image and video communications, signal processing, and machine vision.

Dr. Havlicek was a recipient of the Department of the Navy Award of Merit for Group Achievement in 1990, and he was winner of the 1992 University of Texas Engineering Foundation Award for Exemplary Engineering Teaching while Pursuing a Graduate Degree. He is a member of Tau Beta Pi and Phi Kappa Phi.

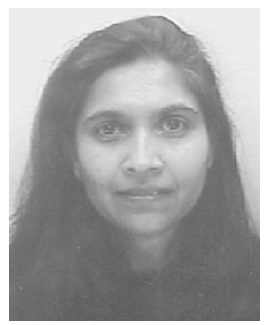

Mita D. Desai (SM'94) received the B.S. degree in electrical engineering from Tennessee State University, Nashville, in 1979 and the M.S. and Ph.D degrees in electrical engineering in 1981 and 1985, respectively, from the University of Illinois, UrbanaChampaign.

From 1989 to 1992, she was a Member of the Technical Staff at the Massachusetts Institute of Technology Lincoln Laboratory, Lexington. Prior to that, she was Principal Research Scientist at Honeywell Systems and Research Center, Minneapolis, MN, from 1985 to 1989. In 1992, she joined the Division of Engineering, University of Texas at San Antonio, where she is currently an Associate Professor. Her current research interests include digital signal and image processing, synthetic aperture radar, biomedical image processing, and 3-D computer vision. She has published over a dozen technical articles in these areas.

Dr. Desai was the General Chair of the 1996 Southwest Symposium on Image Analysis and Interpretation, San Antonio, TX, and is currently an Associate Editor of the IEEE TRANSACTIONS ON IMAGE PROCESSING, and Associate Editor of the international journal Pattern Recognition.

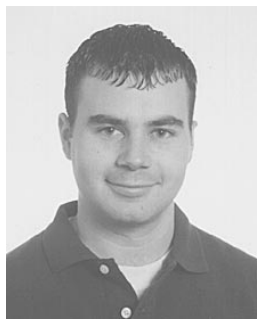

David S. Harding (S'95) was born on August 5, 1974 in Houston, TX. He received the B.S.E. degree with high honors from the University of Texas (UT), Austin in 1995.

He has previously been employed by the Defense Systems and Electronics Group of Texas Instruments, Inc., Dallas, TX, and is currently pursuing the M.S. and Ph.D. degrees at the University of Texas at Austin. Currently, his research interests include nonstationary signal analysis, multidimensional signal processing, and computational vision.

Mr. Harding is a recipient of the UT Austin Microelectronics and Computer Development Fellowship and the Virginia and Ernest Cockrell, Jr. Fellowship in Engineering. 